\title{
The Surroundings of Gamma-Ray Bursts: Constraints on Progenitors
}

\author{
Roger A. Chevalier \\ Department of Astronomy, University of Virginia, P.O. Box 3818, \\ Charlottesville, VA 22903, USA rac5x@virginia.edu
}

\begin{abstract}
Summary. The association of a supernova with a gamma-ray burst (GRB 030329) implies a massive star progenitor, which is expected to have an environment formed by pre-burst stellar winds. Although some sources are consistent with the expected wind environment, many are not, being better fit by a uniform density environment. One possibility is that this is a shocked wind, close to the burst because of a high interstellar pressure and a low mass loss density. Alternatively, there is more than one kind of burst progenitor, some of which interact directly with the interstellar medium. Another proposed environment is a pulsar wind bubble that has expanded inside a supernova, which requires that the supernova precede the burst.
\end{abstract}

\section{Introduction}

Some of the best evidence for nature of gamma-ray burst (GRB) progenitors has come from the identification of the Type Ic supernovae SN 1998bw with GRB 980425 18 and of the recent SN 2003dh with GRB 030329 [49. The finding of these events supports models of long-duration GRBs originating from stripped massive stars [35. The surroundings of massive stars are expected to be shaped by the winds emanating from the progenitor stars. Clear evidence for the signature of a wind has been difficult to establish, and the possibility remains that there is more than one type of progenitor for the long-duration bursts. Direct interaction with the interstellar medium might be expected if the progenitor involves a compact binary system.

Another possibility is that the GRB occurs in a massive star weeks to years after it has become a supernova [51. The progenitor of the GRB may be a rapidly spinning neutron star that spins down and eventually collapses, leading to a burst. This can create a pulsar wind nebula immediately surrounding the burst progenitor, which has possible advantages for producing a GRB 28]. 


\section{Afterglows}

\subsection{Afterglows and the circumburst medium}

The afterglows of GRBs provide a probe of the immediate surroundings of GRBs. The evidence that we have from bursts related to supernovae is that the progenitors are Type Ic supernovae. In addition to SN 1998bw and SN 2003dh, the burst GRB 021211 has possibly been identified with a Type Ic supernova like SN 1994I [12]. These supernovae are thought to have massive star progenitors that have been stripped of their hydrogen envelopes, i.e. WolfRayet stars. This type of massive star progenitor is also suggested by the argument that the collimated flow from a burst be able to pass through the star in a time that is not significantly longer that the duration of the GRB. Even for the long duration bursts, this implies a relatively compact stellar progenitor like the Wolf-Rayet stars 35, 37.

Wolf-Rayet stars are found to have winds with typical mass loss rate $\dot{M}=$ $10^{-5} M_{\odot} \mathrm{yr}^{-1}$ and wind velocity $v_{w}=1000 \mathrm{~km} \mathrm{~s}^{-1}$ ending in a termination shock where the wind runs into the surrounding medium [6]. A steady wind produces a density distribution $\rho=A r^{-2}$; the value of the density can be scaled to the corresponding value for the standard wind parameters, $A_{*}=$ $A /\left(5 \times 10^{11} \mathrm{gm} \mathrm{cm}^{-1}\right)$. For a surrounding medium of pressure $p$, the wind termination shock occurs at a radius

$$
R_{t}=5.7 \times 10^{19}\left(\frac{v_{w}}{1000 \mathrm{~km} \mathrm{~s}^{-1}}\right)\left(\frac{p / k}{10^{4} \mathrm{~cm}^{-3} \mathrm{~K}}\right)^{-1 / 2} A_{*}^{1 / 2} \mathrm{~cm}
$$

where $k$ is Boltzmann's constant. The pressure is normalized to a typical value in the interstellar medium of our Galaxy. A higher pressure can occur as a result of the wind bubble evolution, or as the result of an especially high pressure interstellar medium.

For a spherical explosion with energy $E$, the blast wave in the free wind reaches the radius $R_{t}$ after a time

$$
t_{t}=1.35 \times 10^{4}\left(\frac{v_{w}}{1000 \mathrm{~km} \mathrm{~s}^{-1}}\right)^{2}\left(\frac{p / k}{10^{4} \mathrm{~cm}^{-3} \mathrm{~K}}\right)^{-1}\left(\frac{E}{10^{53} \mathrm{ergs}}\right)^{-1} A_{*}^{2} \text { days. }
$$

This shows that over typical times of observation, the blast wave may be expanding into the free wind for standard parameters. In view of this, interaction with a wind has become one of the models that is investigated in modeling the afterglows of GRBs [7]. This model is compared to results for a constant density medium, which was initially taken in afterglow modeling as the simplest assumption. Taking the surrounding density of the form $\rho \propto r^{-s}$, these cases can be designated $s=0$ (uniform) and $s=2$ (wind). In afterglow models, both wind and constant density models provide adequate fits to the data in some cases, although the constant density model is usually favored [1], 42]. 
At first sight, this ambiguity is surprising. In the context of models with constant efficiencies evolving before a jet break sets in, the two cases have distinctive behavior: the synchrotron self-absorption frequency, $\nu_{a}$, evolves to higher frequency with $s=2$, but remains constant for $s=0$; the peak flux drops with time for $s=2$, but remains constant for $s=0$; the synchrotron cooling frequency, $\nu_{c}$, evolves to higher frequency for $s=2$, but evolves to lower frequency for $s=0$. There are various reasons why these differences have not provided clear tests of the models. The evolution of $\nu_{c}$ requires good light curve information at optical/IR and X-ray wavelengths, which is usually lacking. The evolution of $\nu_{a}$ requires early radio data; these data are usually sparse and are affected by interstellar scintillation. Another problem is that jet breaks are observed in light curves and the evolution in the post-break regime can mimic some of the features of evolution in an $s=2$ medium: the peak flux drops with time and $\nu_{a}$ evolves to higher frequency [45].

Another possibility for distinguishing between the models is to go to very early times, within about a minute of the GRB burst. In addition to the older case of GRB 990123, this has recently been achieved for GRB 021004 [13] and GRB 021211 32, 53, 43. The advantage of these early times is that jet effects do not play a role in the evolution. Li \& Chevalier [34 suggested that the early flat optical light curve of GRB 021004 could be interpreted in terms of wind interaction, in which the critical frequency $\nu_{m}$ had not yet moved down through optical wavelengths. Although this model has some promise, the case remains ambiguous in that the relatively flat evolution might also be produced by a combination of emission from the reverse shock wave and the later forward shock emission in the case of interaction with a $s=0$ medium 27. To distinguish between these possibilities, both good light curve data and color information are needed.

An additional problem with GRB 021004 is that the optical light curve showed variability superposed on the overall trend 25]. This makes it difficult to clearly specify characteristic times in the evolution of the afterglow. The variability, which is not seen in all afterglows, may be due to density inhomogeneities in the circumburst medium and may thus provide additional diagnostics for that medium [24. The winds of Wolf-Rayet stars are known to be inhomogeneous, with clumps filling $\sim 1 / 4$ of the volume [22]. However, the observed degree of inhomogeneity refers to a region close to the stellar surface and the inhomogeneity may decrease in the outer parts of the wind where the afterglow occurs.

\subsection{Jets}

Jet breaks themselves provide a possible diagnostic for the medium. The usual assumption has been that an afterglow light curve steepens due to geometric and spreading effects when its Lorentz factor is about $1 /$ (opening angle) to the form $t^{-p}$ [45]. Kumar \& Panaitescu [29] find that the transition to the asymptotic jet evolution requires a factor $\gtrsim 10$ in time for expansion in a 
uniform medium, but expansion by $\sim 10^{4}$ in time for expansion in a wind medium. However, they used a simplified treatment of jet evolution. Numerical simulations indicate that sharp jet breaks do occur in a uniform medium, but that most of the emission remains within the initial opening angle of the jet 20]. Granot \& Kumar [19] have recently considered structured jets in uniform and declining density media, and again found that jets in an $s=2$ medium cannot give sharp jet breaks.

The recent burst GRB 030329, which was clearly associated with a supernova, showed a sharp break in the light curve at $t \sim 0.5$ day, which has been interpreted as a jet break 3. However, it has become clear that there is considerable structure in the light curve of GRB 030329 over the first 10 days [50, 36] and the identification of an early jet break cannot be made with certainty.

\subsection{Afterglow parameters}

There are uncertainties in the basic assumptions involved in standard afterglow modeling, which include constant values of electron energy efficiency, $\epsilon_{e}$, magnetic energy efficiency, $\epsilon_{B}$, and particle spectral index, $p$, in the evolution of one source (e.g., 46]). If these parameters remain constant during the evolution of one source, the expectation would be that they tend toward "universal" values that apply to various sources. Standard models developed for observed afterglows do not show this. As an example, I take the results of Panaitescu \& Kumar [42, who treat a set of the 10 best observed afterglows with the standard assumptions. The values of spectral index, $p$, cover the range $1.36-2.78$. The presence of values $p<2$ is noteworthy because most of the particle energy is at high energy for this case, although the number of particles is dominated by the low energy particles. The values of $\epsilon_{B}$ and $\epsilon_{e}$ cover the ranges of $4 \times 10^{-5}-0.07$ and $0.01-0.4$, respectively.

The theoretical values of these parameters are poorly known. The production of the magnetic field requires some mechanism at the forward shock front to build up the magnetic field. The mechanism remains uncertain, although the Weibel instability has been suggested 38, and recent simulations show some promise for this mechanism [40, 16]. Studies of Fermi-type particle acceleration in ultarelativistic shocks have yielded a preferred value of $p=2.2-2.3$ in the test particle limit [1]. The way electrons are injected into the acceleration process remains uncertain, and there are indications that the acceleration may differ from the Fermi process [40, 16.

The fact that a range of parameters is needed to explain the various afterglows suggests that the parameters depend on the physical conditions. It might be expected that the shock velocity and preshock density are important determinants of the physical conditions, so that the model parameters should vary during the evolution of a burst. Yost et al. [54 have recently considered models in which $\epsilon_{B}$ is allowed to vary as a power law of the shock Lorentz factor and in which the value of the preshock density parameter $s$ is allowed to 
cover a large range. Their modeling of 4 sources shows that a wide variety of models are potentially possible, including ones in which the density increases steeply with radius. It appears that more extensive observations, including spectral information over a wide time range, are needed to further constrain the models. One possibility is to follow the evolution of the characteristic frequency, $\nu_{m}$, from optical to radio wavelengths. This frequency is typically observed at radio wavelengths on a timescale $\sim 10$ days. Its passage at optical wavelengths requires very early observations; as noted above, this may have been observed in GRB 021004. The typical frequency is not sensitive to the density, but it is sensitive to the efficiency factors, so that constraints on their evolution may be obtainable.

\subsection{A shocked wind environment}

In view of the evidence for the association of GRBs with massive stars and the evidence from afterglows for interaction with a constant density medium, consideration must be given as to how a massive star might produce a uniform density surrounding. The most plausible way to do this is the approximately constant density region expected downstream from the wind termination shock [52. Wijers 52 suggested two ways for creating a smaller value of $t_{t}$ (eq. 22): reducing the mass loss rate from the progenitor to $10^{-6} M_{\odot} \mathrm{yr}^{-1}$ because of the low metallicity of the progenitor star and increasing the pressure by interacting with dense molecular gas, especially if the progenitor star is moving. However, the metallicity dependence of the mass loss from Wolf-Rayet stars is uncertain: WN type stars in the lower metallicity Large Magellanic Cloud 23] and the Small Magellanic Cloud 9] have similar mass loss rates to those in the Galaxy, although WC stars do seem to show a metallicity effect [10. Also, photoionizing radiation during the life of a massive star tends to clear a region around the star to a moderately low density. Another way of increasing $p$ is by having the burst occur in a high pressure starburst region [15], where the pressure can reach values of $p / k \gtrsim 10^{8} \mathrm{~cm}^{-3} \mathrm{~K}$ [4]. In this case, there should be a relation between the properties of the afterglow (relatively dense surroundings) and the position of the burst relative to a region of very active star formation.

One expectation of the models with a termination shock is that some bursts should be observed to make a transition from an $s=2$ to an $s=0$ medium, with a density jump between them. There has been little evidence for such a transition. The expectation for such a transition is that the light curve should evolve to a flatter asymptotic decline after a jump in flux. Wijers [52] mentioned GRB 970508 because it had a bump in the optical light curve at an age of 1 day. However, it did not show the expected flattening of the light curve. A burst that showed a steepening with a possible bump is GRB 030226. Dai \& Wu [1] suggested that the transition was due to the interaction with a large density jump, which might occur at the contact discontinuity between the shocked progenitor wind and the dense red supergiant wind from 
a previous evolutionary phase. In this picture, the steepening of the light curve is due to the sideways expansion of the jet in the dense medium. However, the data on GRB 030226 are not of sufficient quality to clearly show the expected features at the time of transition with the density jump. In the case of interaction with the termination shock of the stellar wind, the density jump is such that the effects of the reverse shock are not expected to be important, as opposed to the high density jump case.

\section{Optical/ultraviolet absorption lines}

A recent development relevant to the surroundings of GRBs is the observation of strong optical/ultraviolet absorption lines in a few cases. The best case is GRB 021004, which has a redshift $z=2.32$ so that strong ultraviolet lines in the rest frame are redshifted to optical wavelengths [39, 47. Strong lines of Ly $\alpha, \operatorname{Ly} \beta, \mathrm{C}$ IV, and Si IV are found blueshifted relative to the host velocity by $-450,-990$, and $-3155 \mathrm{~km} \mathrm{~s}^{-1}$ [39. The lines have not been observed to vary, so they cannot be directly tied to the immediate circumburst environment, but both Mirabal et al. 39] and Schaefer et al. [47] argue that they are likely to be formed in the nearby environment. One argument for this is that the strong lines are unusual for intervening systems observed in the spectra of quasars. The lines may be formed close to the host galaxy if the burst occurred in a starburst region with a strong galactic superwind. However, the maximum velocity shift is higher than has been observed in galactic superwinds. In addition, the high velocity would require a high initial temperature for the gas if the wind is thermally driven. The gas would be completely ionized and it is unlikely that it would be able to cool to allow the observed ions.

For a circumstellar origin, there are two possibilities: the high velocities are related to the wind velocities of the progenitor system, or the velocities are due to radiative acceleration by the GRB light; combinations of these models are also possible. Schaefer et al. 47] argue that the high blueshifted velocity can be naturally produced by the Wolf-Rayet star wind velocity and the lower velocity components can be identified with denser shells swept-up by the fast wind. Mirabal et al. 39 argue that the abundances deduced from the lines disfavor the wind model. Hydrogen is present in the observed lines, but it is also observed in the spectra of some WN stars [48]; however, the absence of $\mathrm{N} \mathrm{V}$ lines in the observed spectra indicates that $\mathrm{N}$ is not overabundant. In the radiatively accelerated model, the accelerated clumps must be at an initial distance of several 0.1's pc from the progenitor star. The acceleration, primarily by bound-free transitions, must occur early in order to avoid the observation of time variability.

The problem with both of these scenarios is that the strong radiation field from the GRB and its afterglow is able to completely photoionize the gas out to a distance $\gtrsim 10^{18} \mathrm{~cm}\left[31\right.$. At a sufficiently high density $\left(\gtrsim 10^{7} \mathrm{~cm}^{-3}\right)$, the recombination time becomes shorter than the age of the burst. This density 
might be present in clumps of the swept-up red supergiant wind, especially if the progenitor is in a high pressure region, but further exploration of this topic is needed.

GRB 021004 is not alone in showing these line features. High excitation, high velocity absorption features have been found in GRB 020813 2] and GRB 030226 [21, 44, 8. The absorption lines of CIV in GRB 020813 are at $0 \mathrm{~km} \mathrm{~s}^{-1}$ and $-4320 \mathrm{~km} \mathrm{~s}^{-1}$ relative to the host. In this case, the blueshifted absorption is also present in a number of lower ionization species (Si II, Al II, Fe II, Mg II, and Mg I); there is no coverage of Ly $\alpha$. In the case of GRB 030226, strong absorption line systems are present at a velocity separation of $2300 \mathrm{~km} \mathrm{~s}^{-1}$, with C IV and Si IV present, as well as numerous lower ionization species and Ly $\alpha$. The velocity separation seen in these sources is consistent with expectations for the velocity of a Wolf-Rayet star wind. However, the presence of $\mathrm{H}$ does not support this origin for the lines.

\section{Pulsar wind bubble environment}

An interesting possibility for a GRB environment is that created by a pulsar wind nebula. This possibility was proposed in the context of the supranova model in which the supernova precedes the GRB [51, 28. In this scenario, the supernova core contracts to a massive, rapidly rotating neutron star which spins down and collapses to a black hole after a period of weeks, months, or years. If the neutron star has a magnetic field similar to that of radio pulsars, it can create a shocked bubble of relativistic electron/positron fluid and magnetic field before it collapses. The bubble accelerates the supernova ejecta, so that the ejecta can play a role in producing the X-ray lines that have possibly been observed in some bursts.

One issue is how effectively the pulsar nebula can accelerate the supernova gas, because the situation is subject to Rayleigh-Taylor instabilities [28, 26]. The X-ray line features are typically blueshifted by $\sim 0.1 c$, which is higher than the velocities of the heavy element ejecta that would be expected from the supernova itself. A supernova energy of $10^{51} \mathrm{ergs}$ in an ejecta mass of $10 M_{\odot}$ leads to a typical velocity of $0.01 \mathrm{c}$. Königl \& Granot 28 suggest that the rotational energy of a rapidly rotating neutron star, $10^{53} \mathrm{ergs}$, is transferred to the ejecta, giving the observed velocity. Although a pulsar nebula can certainly shock and compress the ejecta gas, the ability to further accelerate the ejecta is less certain. However, if the supernova explosion energy is high ( $>10^{52}$ ergs as inferred for some supernovae) and the ejecta mass is low, a typical velocity of $0.1 c$ can be attained.

Another issue is the fact that the observation of X-ray line features at an age of $\sim 1$ day requires dense gas at $r \sim 10^{16} \mathrm{~cm}$, but observations of afterglow emission at an age of a week or more imply a radial scale $\gtrsim 3 \times 10^{17}$ cm. Königl \& Granot 28] suggest that pulsar nebula and supernova may be highly elongated along the axis along which the GRB flow propagates. 
Although the observational evidence for X-ray lines remains controversial, there are other reasons for considering a pulsar wind nebula environment, as articulated by Königl \& Granot 28. One is that the pulsar nebula is composed of just the ingredients that are necessary for the synchrotron emission from a GRB afterglow. There is no problem with the efficiencies for production of the synchrotron emission. In addition, the bubble density can be constant with radius, or drop with radius. The first case can occur in the same way that a constant density occurs downstream from an ordinary stellar wind. The decreasing density occurs in regions where the magnetic field pressure becomes important, although the structure of such regions in pulsar nebulae remains uncertain.

Although the pulsar bubble model has some appeal, it does not apply to cases where the GRB occurs close in time to the supernova, as apparently was the case with GRB 030329 and SN 2003dh (e.g., [36]).

\section{Discussion and conclusions}

Despite several years of effort, the study of GRB environments from their interaction has not clearly pointed to the progenitor objects. Reasons for this include the uncertainties in the basic model parameters, the fact that the GRB ejecta appear to be collimated and the similarity in the surrounding densities expected in different scenarios. The clearest progenitor information we have comes from the association of SN 2003dh with GRB 030329. The similarity of the supernova to SN 1998bw suggests that the progenitor object is a similar massive star. Analysis of the radio emission from SN 1998bw showed compatibility with expectations of a wind-like surrounding medium 33. The radio observations of GRB 030329 are slightly better fit by a uniform medium than a wind-like medium, although the difference between the fits is not large 3. The host galaxy of GRB 030329 appears to be a starburst dwarf galaxy [36] and the burst is positioned near the edge of the star forming region [17, so it is not clear whether a high pressure surroundings is expected. Detailed modeling will be needed to determine whether the afterglow features can be explained by a complex explosion, or whether the surroundings need special properties.

An outstanding question is whether there is any need for a progenitor of long-duration bursts other than massive stars. Since massive stars are expected to modify their surroundings through winds, this evidence would be an incompatibility with the wind effects expected around a massive star. Frail et al. 14] and Yost et al. [54] have noted that a number of afterglows that can best be modeled as expanding into a uniform density medium with $n \sim 10-30$

$\mathrm{cm}^{-3}$, and that this density is typical of Galactic interstellar clouds or the interclump medium of molecular clouds. However, such a medium would be modified by the winds from a massive star progenitor and a significant fraction of GRBs probably occur in starburst regions where clouds are denser 
than in Galactic case. One possibility is that the uniform medium is created by a shocked stellar wind in a high pressure medium.

A possible problem for massive star models is the low density inferred around some GRBs. Even if a shocked wind is present, it has higher density at a given radius than the corresponding free wind, so strong limits on the wind density can be set. Two afterglows with low densities are GRB 990123 42] and GRB 021211 [30], which require $A_{*} \lesssim 10^{-3}-10^{-2}$. Such low densities have not observed around Wolf-Rayet stars. GRB 021211 shows evidence for a supernova 12, but the evidence is not conclusive. The low densities are not a problem for a burst that interacts directly with the hot interstellar medium in a galaxy. Another problem with the massive star models is the lack of evidence for bursts crossing the interface between a free wind and a shocked wind.

If direct interaction with the interstellar medium is required, a plausible progenitor object is a binary of compact objects. This requires that two different progenitor objects can give GRBs that appear similar, presumably from the formation of black holes. The application of the pulsar wind nebula model to some bursts requires both that different progenitor objects give rise to similar bursts, but also that the expansion of the burst into a different kind of medium (pair plasma and magnetic field) can give rise to similar afterglows. The finding of a supernova (SN 2003dh) occurring at approximately the same time as a GRB (GRB 030329) mitigates against the pulsar wind nebula picture for this case.

I am grateful to Jon Marcaide and the other conference organizers for their hospitality in Valencia, and to Zhi-Yun Li and Claes Fransson for continuing collaboration on these topics. This work was supported in part by NASA grant NAG5-13272 and NSF grant AST-0307366.

\section{References}

1. A. Achterberg, Y.A. Gallant, J.G. Kirk, A.W. Guthmann: MNRAS 328, 393 (2001)

2. A.J. Barth, et al.: ApJ, 584, L47

3. E. Berger, et al.: Nature, in press (astro-ph/0308187) (2003)

4. R.A. Chevalier, C. Fransson: ApJ 558, L27 (2001)

5. R.A. Chevalier, J.N. Imamura: ApJ 270, 554 (1983)

6. R.A. Chevalier, Z.-Y. Li: ApJ 520, L29 (1999)

7. R.A. Chevalier, Z.-Y. Li: ApJ 536, 195 (2000)

8. R. Chornock, A.V. Filippenko: GCN 1897 (2003)

9. P.A. Crowther: A\&A, 356, 191 (2000)

10. P.A. Crowther, L. Dessart, D.J. Hillier, J.B. Abbott, A.W Fullerton: A\&A, 392, 653 (2002)

11. Z.G. Dai, X.F. Wu: ApJ 591, L21 (2003)

12. M. Della Valle, et al.: A\&A 406, L33 (2003)

13. D.W. Fox et al: Nature 422, 284 (2003)

14. D.A. Frail, et al.: ApJ 590, 992 (2003) 
15. C. Fransson: These proceedings (2003)

16. J.T. Frederikson, C.B. Hededal, T. Haugboelle, A. Nordlund: ApJ, submitted (astro-ph/0308104) (2003)

17. A. Fruchter, et al.: GCN 2243 (2003)

18. T.J. Galama, et al.: Nature, 395, 670 (1998)

19. J. Granot, P. Kumar: ApJ 591, 1086 (2003)

20. J. Granot, M. Miller, T. Piran, W.-M. Suen, P.A. Hughes. In: Gamma-Ray Bursts in the Afterglow Era, ed. by E. Costa, F. Frontera, J. Hjorth (Springer: Berlin), 312 (2001)

21. J. Greiner, E. Guenther, S. Klose, R. Schwarz: GCN 1886 (2003)

22. W.-R. Hamann, L. Koesterke: A\&A 335, 1003 (1998)

23. W.-R. Hamann, L. Koesterke: A\&A 360, 647 (2000)

24. J.S. Heyl, R. Perna: ApJ 586, L13 (2003)

25. S.T. Holland et al.: AJ 125, 2291 (2003)

26. S. Inoue, D. Guetta, F. Pacini: ApJ 583, 379 (2003)

27. S. Kobayashi, B. Zhang: ApJ 582, L75 (2003)

28. A. Königl, J. Granot: ApJ 574, 134 (2002)

29. P. Kumar, A. Panaitescu: ApJ 541, L9 (2000)

30. P. Kumar, A. Panaitescu: preprint (astro-ph/0305446) (2003)

31. D. Lazzati, E, Rossi, S. Covino, G. Ghisellini, D, Malesani: A\&A 396, L5 (2002)

32. W. Li, A.V. Filippenko, R. Chornock, S. Jha: ApJ 586, L9 (2003)

33. Z.-Y. Li, R.A. Chevalier: ApJ 526, 716 (1999)

34. Z.-Y. Li, R.A. Chevalier: ApJ 589, L69 (2003)

35. A.I. MacFadyen, S.E. Woosley, A. Heger: ApJ 550, 410 (2001)

36. T. Matheson, et al.: ApJ, in press (astro-ph/0307435) (2003)

37. C.D. Matzner: MNRAS, in press (astro-ph/0203085) (2003)

38. M.V. Medvedev, A. Loeb: ApJ 526, 697 (1999)

39. N. Mirabal, et al.: ApJ, in press (astro-ph/0303616) (2003)

40. K.-I. Nishikawa, P. Hardee, G. Richardson, R. Preece, H. Sol, G.J. Fishman: ApJ, in press (astro-ph/0305091) (2003)

41. A. Panaitescu, P. Kumar: ApJ 554, 667 (2001)

42. A. Panaitescu, P. Kumar: ApJ, 571, 779 (2002)

43. H.S. Park, G. Williams, S. Barthelmy: GCN 1736 (2002)

44. P.A. Price, D.W. Fox, S.G. Djorgovski, P. Cote, A. Jordan: GCN 1889, (2003)

45. R. Sari, T. Piran, J.P. Halpern: ApJ 519, L17 (1999)

46. R. Sari, T. Piran, R. Narayan: ApJ 497, L17 (1998)

47. B.E. Schaefer, et al.: ApJ 588, 387 (2003)

48. L.F. Smith, M.M. Shara, A.F.J. Moffat: MNRAS 281, 163 (1996)

49. K.Z. Stanek, et al.: ApJ, 591, L17 (2003)

50. M. Uemura, et al.: Nature 423, 843 (2003)

51. M. Vietri, L. Stella: ApJ 507, L45 (1998)

52. R.A.M.J. Wijers. In: Gamma-Ray Bursts in the Afterglow Era, ed. by E. Costa, F. Frontera, J. Hjorth (Springer: Berlin), 306 (2001)

53. P. Wozniak, et al.: GCN 1757 (2002)

54. S.A. Yost, F.A. Harrison, R. Sari, D.A. Frail: ApJ, submitted (astroph/0307056) (2003) 